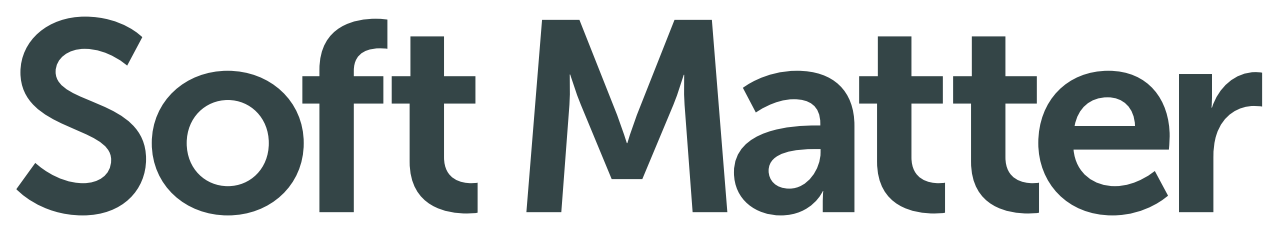


Check for updates

Cite this: Soft Matter, 2019, 15,4629

Received 14th February 2019, Accepted 7th May 2019

DOI: $10.1039 / \mathrm{c} 9 \mathrm{sm} 00318 \mathrm{e}$

rsc.li/soft-matter-journal

\title{
Deep pool water-impacts of viscous oil droplets $\dagger$
}

\author{
Utkarsh Jain, (D) *a Maziyar Jalaal, (D) ${ }^{a}$ Detlef Lohse (iD) ab and \\ Devaraj van der Meer (iD ${ }^{a}$
}

\begin{abstract}
We experimentally study the impacts of viscous, immiscible oil drops into a deep pool of water. Within the target liquid pool, the impacting drop creates a crater, whose dynamics are studied. It is found that the inertia of pool liquid and drop viscosity are the main factors that determine the crater's maximum depth, while the additional factor of mutual immiscibility between the drop and pool liquids leads to interesting interfacial dynamics along the oil-water interface. We discuss how this can change the crater dynamics in its retraction phase, making possible a type of double-entrainment, whereby a tiny air bubble is entrapped inside a water-entrained oil drop. Further, we report the observation of a type of 'fingering' that occurs along the oil-drop rim, which we discuss, arises as a remnant of the well-known crown-splash instability.
\end{abstract}

\section{Introduction}

Drop impacts on a deep pool of liquid ${ }^{1-6}$ and on different types of solid substrates ${ }^{7-9}$ have been an active subject of research for a long time. This finds its place in a wider tradition of the work on water entry comprehensively covered by Truscott et al. ${ }^{10}$ Drop impact studies differ from solid projectile impacts in that, in the former, the impacting projectile is deformable, which has also become of interest as evidenced by recent works of Hurd et al. ${ }^{11}$ and Jalaal et al. ${ }^{12}$

Upon the impact of a drop on a deep liquid pool, a crater is formed below the free surface of the target, whose collapse can result in phenomena such as bubble entrainment and formation of a Worthington jet, depending on the physical conditions. ${ }^{6}$ To understand these common features, which constitute fundamental questions about cavity formation, collapse and jet formation, the experimental focus has been almost exclusively on impacts where the drop and pool consist of the same liquid. Having the drop impact on the same liquid makes the problem more tractable, and makes it possible to draw general conclusions about the above mentioned features. However, a more complex situation can exist in some natural processes and industrial applications, such as impacts of drops of different liquids. This is the focus of the present work. A real-world example of a viscous immiscible liquid impacting into water is connected to man-made accidents like oil

\footnotetext{
${ }^{a}$ Physics of Fluids Group and Max Planck Center Twente for Complex Fluid Dynamics, MESA+ Institute and J. M. Burgers Centre for Fluid Dynamics, University of Twente, P.O. Box 217, 7500AE Enschede, The Netherlands. E-mail: u.jain@utwente.nl,m.jalaal@utwente.nl

${ }^{b}$ Max Planck Institute for Dynamics and Self-Organization, Am Fassberg 17, 37077 Göttingen, Germany

$\dagger$ Electronic supplementary information (ESI) available. See DOI: 10.1039/c9sm00318e
}

spill from tankers. An investigation along this line was peformed by Murphy et al. ${ }^{13}$ studying rain drop impacts on a deep pool of seawater covered by a thin oil layer.

Another example of naturally occurring accidents relevant to the present study can be oil blowouts from ocean surface. ${ }^{14}$ In particular, the impinging liquid (here, a drop) could be more viscous than the target liquid, which changes the types of underwater craters and their dynamics. Impacts of viscous, but miscible drops into water have been studied by Walker et al. ${ }^{15}$ and Sharma et al. ${ }^{16}$ where glycerol was used as drop liquid. However, since glycerol is miscible with water, the two liquids undergo mixing during and after impact.

There has been surprisingly little work studying oil-water impacts. Some notable exceptions studying impacts on deep pool are the work by Lhuissier et al. ${ }^{17}$ which principally studies the fragmentation of a water drop impacting silicone oil bath, and the work of Fujimatsu et al. ${ }^{18}$ which also studies water drop impact on oil bulk. The latter focuses on more traditional quantities of interest such as maximum diameter of drop spreading, and depths of the craters formed. More recent works by Che \& Matar ${ }^{19}$ and Shaikh et al. ${ }^{20}$ have studied immiscible drop impacts, but on thin films.

Here, we use silicone oils as drop liquid, which are immiscible with water, and, in contrast to glycerol drops, maintain their bulk and surface properties throughout the impact process. This allows us to use a range of viscosities in the drop liquid, while keeping its surface tension and density nearly constant. We discuss the different crater formation and collapse processes in this configuration, and how it differs from a water drop impacting on water. The oil drop creates a crater in the water pool and spreads over its floor. This creates an extra liquid-liquid interface, which depending on the extent of viscous dissipation in the drop, 
can stretch over a small portion of, or over the entirety of the crater surface. We find that this can have interesting consequences for oil-drop entrainment, and also double-entrainment of an air bubble in the entrapped oil drop. The crater dynamics are further discussed quantitatively and its depth is compared against the predictions of a model based on flow within the target pool.

The paper is organised as follows: in Section 2 we discuss our experimental setup, the procedures followed and the experimental challenges. In Section 3.1, we discuss our observations of crater formation and collapse, and how they differ between oil-drop and water-drop impacting on water pool. The interaction between new oil-water interface and capillary waves during crater collapse leads to interesting types of entrainment, discussed in Section 3.2. Section 4 focusses on crater depth, discussing its dependence on crater potential energy and drop viscosity. Finally we report our observation of fingering along the drop rim in Section 5, followed by the conclusions in Section 6 .

\section{Experimental setup and range of parameters explored}

The setup is shown in Fig. 1. It consists of a syringe pump, which is connected to a nozzle via thin tubing. Nozzles with different diameters were used to generate water and silicone oil drops of varying sizes. The syringe pump was operated at a quasi-static rate to ensure that the droplet which is formed at the opening of a nozzle gets detached by its own weight, and is not affected by the flow-rate of the liquid in the tubing. The generated drop, which is in the range of $3.1-4.4 \mathrm{~mm}$ in diameter, is then allowed to fall into a deep pool of stationary water which is $140 \times 140 \times 70 \mathrm{~mm}^{3}$ in volume. The subsequent events are recorded using high speed cameras (Photron Mini UX 100, 4-5 K fps) from a side to study the crater dynamics

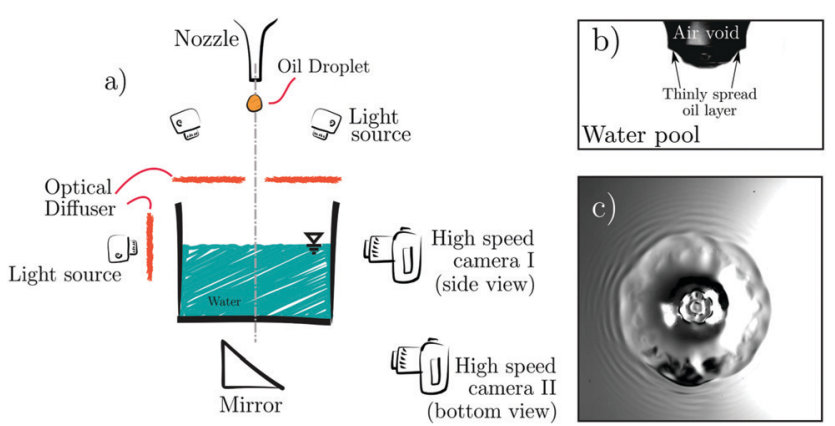

Fig. 1 A schematic of the experimental setup is shown in panel a. Examples of side and bottom-view images are shown in panels $b$ and $c$ respectively.
(Sections 3.1 and 4), and from the bottom (Photron SAX, 10-12 $\mathrm{K} \mathrm{fps)} \mathrm{to} \mathrm{study} \mathrm{the} \mathrm{detailed} \mathrm{evolution} \mathrm{of} \mathrm{the} \mathrm{oil-water}$ interface (Section 5). The imaging from the side was done by the conventional means of using a brightly lit diffuser plate, which serves as the background for recording sub-surface crater behaviour. Imaging from the bottom similarly required a light diffuser. However, simply placing a diffuser in the optical path of the bottom-view camera would obstruct the falling drop in its path. Thus, light diffusion was achieved by using sheets of tracing paper, into which a hole was made to allow for the passage of falling drops. Cold light sources (Olympus ILP-1 and Sumita LS-M352A) were used to avoid heating the water in the target pool. All experiments were conducted at standard room temperature of $20 \pm 0.5{ }^{\circ} \mathrm{C}$. The liquids listed in Table 1 were used throughout the present study.

Since the main drop detaches by its own weight and forms via a Rayleigh-Plateau instability, it is often accompanied by the creation of smaller satellite drops. It was observed that the number of satellite drops produced was greater for oils of smaller viscosity. In some experiments, the smaller drops would follow the main drop in impacting on the stationary water pool and interfere with the crater dynamics. Thus, only those experiments were analysed in which the satellite drops were either not produced at all, or trailed far enough behind the main drop such as to not interfere with the crater dynamics of interest. After each experiment, the oil drop was observed to rapidly spread all over the pool liquid surface due to its low interfacial tension with water. ${ }^{21}$ This contaminated both the target pool surface, and the container walls. As a result, each experiment required the container walls to be thoroughly cleaned, and the whole pool liquid to be replaced with a fresh volume of water. Both these reasons - the requirement of thorough cleaning of the target liquid containers, and the production of satellite drops, limited the number of experiments that could be conducted. The relevant non-dimensional numbers, Weber, Froude and Reynolds are defined as

$$
\mathrm{We}=\frac{\rho_{\mathrm{d}} v^{2} D_{0}}{\sigma_{\mathrm{d}}}, \quad \mathrm{Re}=\frac{\rho_{\mathrm{d}} v D_{0}}{\mu_{\mathrm{d}}}, \quad \text { and } \quad \mathrm{Fr}=\frac{v^{2}}{g D_{0}},
$$

where, $\rho$ is the density, $v$ the drop velocity, $\sigma$ the surface tension, $\mu$ the viscosity, and the subscript $d$ is used for drop characteristics, while $D_{0}$ is the diameter of the impinging drop. We and Re can also be combined via Capillary number defined as

$$
\mathrm{Ca}=\frac{\mathrm{We}}{\mathrm{Re}}=\frac{\mu v}{\sigma} \text {. }
$$

The density and surface tension ratios between the drop-pool liquids remain at approximately constant values of $0.963-0.97$ and 0.29 , respectively (see Table 1 ) for all oil drop impact experiments,

\begin{tabular}{|c|c|c|c|c|}
\hline Fluids & Viscosity $\mu$ (Pa s) & Density $\rho\left(\mathrm{kg} \mathrm{m}^{-3}\right)$ & Surface tension $\sigma\left(\mathrm{mN} \mathrm{m}^{-1}\right)$ & Manufacturer \\
\hline Water & $0.89 \times 10^{-3}$ & 1000 & 72 & Milli-Q \\
\hline Silicone oil $35 \mathrm{cSt}$ & $30 \times 10^{-3}$ & 963 & 21 & Wacker Chemie AG \\
\hline Silicone oil $50 \mathrm{cSt}$ & $42.9 \times 10^{-3}$ & 963 & 21 & Wacker Chemie AG \\
\hline Silicone oil $100 \mathrm{cSt}$ & $85.9 \times 10^{-3}$ & 965 & 21 & Wacker Chemie AG \\
\hline Silicone oil $200 \mathrm{cSt}$ & $172.7 \times 10^{-3}$ & 970 & 21 & Wacker Chemie AG \\
\hline
\end{tabular}

Table 1 Fluids used in the present experiments and their properties (at $25^{\circ} \mathrm{C}$ ) sourced from supplier data sheets 
while the viscosity ratio between the drop liquid and water: $\mu_{\mathrm{d}} / \mu_{\mathrm{w}}$ varies in the range 33.7-193.3. In the present study, a major focus is on the crater formation process after drop impact. Since crater formation is mainly an inertial process, for which the drop size sets the length scale, we choose the drop diameter $D_{0}$ as the representative length scale in all the experiments. Throughout the study, the target pool consisted of purified water, whereas the drop liquid was changed to study the effect of drop viscosity on crater dynamics. Thus, it was deemed suitable to use the drop liquid viscosities to define the Reynolds number.

\section{Qualitative description of the experimental results}

\subsection{Crater formation and collapse}

Qualitative examples of a drop of water and silicone oils with different viscosities impacting onto a deep pool of water are shown in Fig. 2 (all with $\mathrm{Fr} \approx 180$ ) and 3 (all with $\mathrm{Fr} \approx 300$ ). A water drop impacting into a water pool penetrates the target pool and, in the initial stages, creates a bucket-shaped crater with steep walls (see Fig. 2a). The crater then rapidly grows into a roughly hemispherical shape and expands radially. These observations have been made since the earliest water dropimpact studies. ${ }^{4,22}$ An impacting drop of viscous oil, however, creates a notably different crater in the initial stages of impact. Instead of a flat bottomed crater where the pool liquid is exposed to air, we observe the crater bottom to have an oil-water interface, which has a slightly greater curvature than the latter from the earliest stages. For instance, see the early stages in Fig. 2b-e where a portion of an impacting viscous oil drop can be seen collected at the bottom of the crater. As the crater expands, the oil drop gets stretched out over the crater surface. It may cover a varying portion of the crater surface depending on its viscosity and the initial impact velocity. Soon after the initial stages of crater formation, the radial expansion of the crater and the downwards motion of the drop set a flow in the pool liquid, which was first reported by Engel $^{4}$ based on observations of water-drop impacts on water pool. This flow in the target pool was understood to result from radial and tangential velocities of the pool liquid, and was used by Bisighini et $a .^{23}$ to propose a potential flow model. It is seen that in the case of both - water drops impacting on water, and oil drops impacting on water with high Weber numbers (We $>$ 1400 and $\mathrm{Re}>100$, Fig. $3 \mathrm{a}$ and $\mathrm{b}$ ), the growing craters are found to be roughly hemispherical in shape for the most part of their expansion phase. Thus, flow in the pool, regardless of the drop liquid, is similar while there is an expanding hemispherical crater.

The impacting drop's energy can be used towards the creation of several phenomena such as the appearance of a crown splash, a wave swell that travels along the pool surface, and a sub-surface crater. Liow ${ }^{24}$ estimated that almost a third of the impacting drop's kinetic energy is converted to the crater's gravitational potential energy. This suggests that a significant portion of the drop energy is spent in other contemporary processes such as the
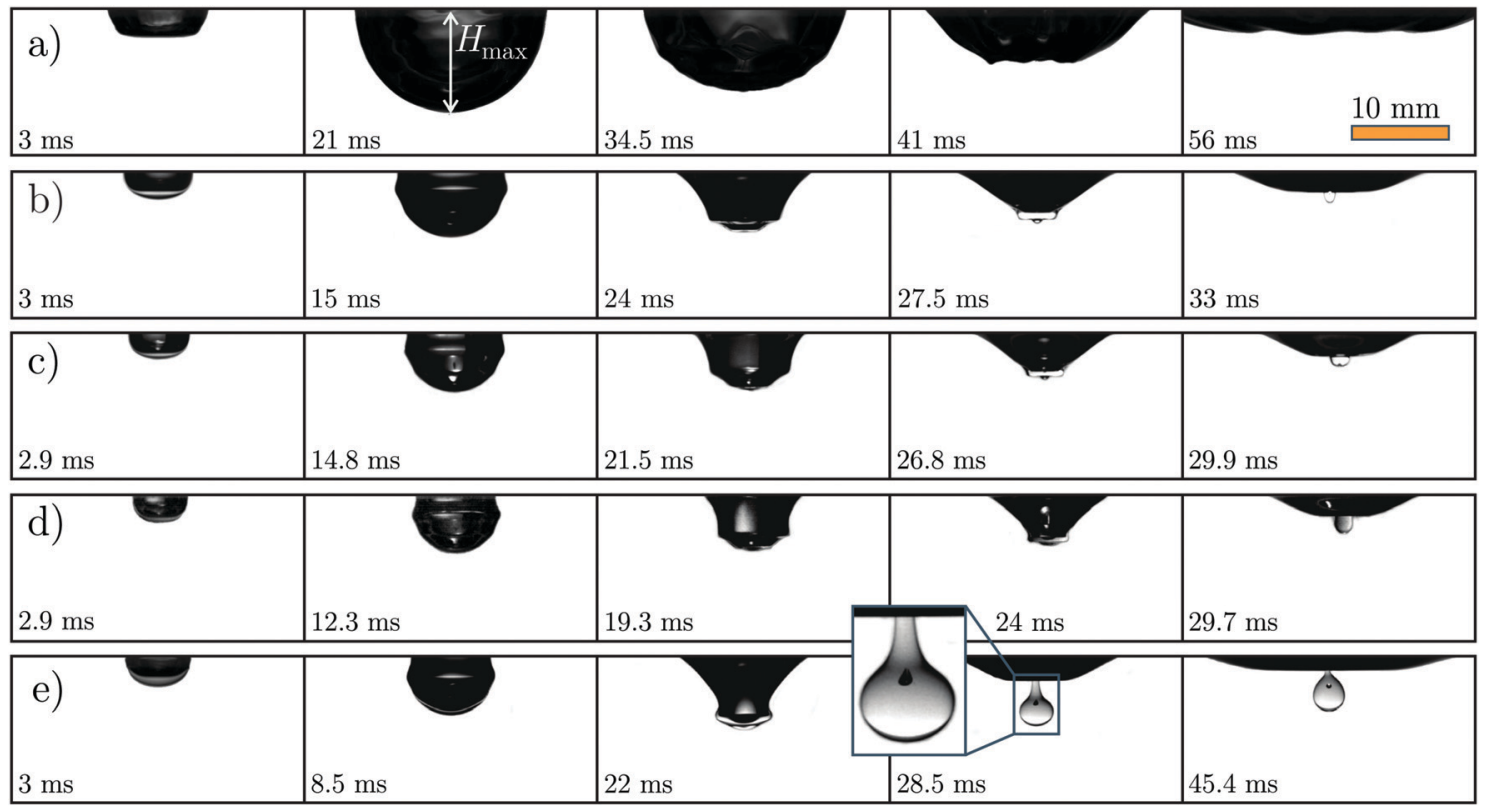

Fig. 2 Typical sub-surface events after the deep-pool water impact of (a) Water drop with $\mathrm{Fr}=168$, We $=444, \mathrm{Re}=13333$, corresponding to Ca $=0.033$, (b) 35 cSt silicone oil drop with $\mathrm{Fr}=178, \mathrm{We}=841, \mathrm{Re}=220$, corresponding to $\mathrm{Ca}=3.82$, (c) $50 \mathrm{cSt}$ silicone oil drop with $\mathrm{Fr}=187, \mathrm{We}=868, \mathrm{Re}=156$, corresponding to $\mathrm{Ca}=5.56$, (d) $100 \mathrm{cSt}$ silicone oil drop with $\mathrm{Fr}=172$, We $=822$, $\mathrm{Re}=76$, corresponding to $\mathrm{Ca}=10.81$ and (e) $200 \mathrm{cSt}$ silicone oil drop with $\mathrm{Fr}=177, \mathrm{We}=829, \mathrm{Re}=38$, which corresponds to $\mathrm{Ca}=21.82$. A closer look at the experiment in panel e reveals an air-bubble entrained in the poolentrained oil drop. See ESI† for the experimental video, and the main text for discussion. All the experiments shown above have comparable Froude numbers of $\approx 180$. Despite this, there is a significant difference in the crater sizes between experiment (a) and experiments (b-e), which can be attributed to different sizes of the impinging drop. This difference in sizes of the impinging drop comes from how the drop is generated via a Rayleigh-Plateau instability. Since $\sigma_{\text {water }}$ is much larger than $\sigma_{\text {silicone oil, }}$ the drops produced are larger in size. 

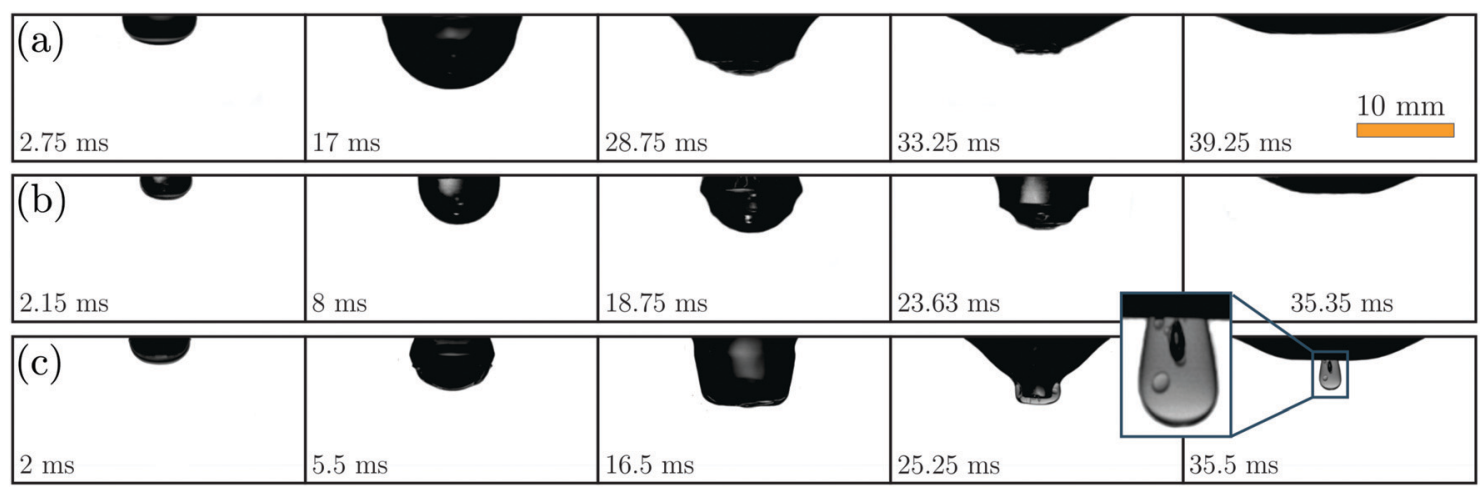

Fig. 3 Typical sub-surface events after the deep-pool impact of (a) $35 \mathrm{cSt}$ silicone oil with $\mathrm{Fr}=287, \mathrm{We}=1502$, $\mathrm{Re}=302$, corresponding to Ca $=4.97$, (b) $100 \mathrm{cSt}$ silicone oil with $\mathrm{Fr}=306, \mathrm{We}=1490, \mathrm{Re}=103$, corresponding to $\mathrm{Ca}=14.47$, (c) $200 \mathrm{cSt}$ silicone oil with $\mathrm{Fr}=312, \mathrm{We}=1424, \mathrm{Re}=50$, corresponding to $\mathrm{Ca}=28.48$. All the experiments shown above have comparable $\mathrm{Fr} \approx 300$. As in Fig. 2 , a closer look at the experiment in panel $\mathrm{c}$ reveals an air-bubble entrained in the pool-entrained oil drop.

creation and expansion of new liquid-liquid and liquid-air interfaces, creation and lifting up of a splash-crown, and viscous dissipation in the liquid components. ${ }^{25,26}$

The crater growth is finally arrested mainly by gravitational potential energy of the displaced fluid. ${ }^{4,27}$ Drops impacting with large Froude numbers create large craters, which in turn have large associated potential energy. To conclude, crater potential energy is one of the main energy sinks during the early stages of crater expansion process,$^{28}$ and the main factor behind its subsequent retraction.

\subsection{Crater retraction and entrainment}

In later stages of crater growth, capillary waves appear on the crater walls which start from the raised sheet of liquid and move downwards, eventually focussing at the bottom. The waves travel inwards along the crater walls and deform the crater. Their effect on crater shapes and bubble entrapment in single-phase drop impact have been studied in detail in previous studies. ${ }^{29-33}$ In the experiments shown for oils of viscosity up to $100 \mathrm{cSt}$, the drop liquid was seen to spread over the whole crater surface for the given Froude numbers. Since the crater sizes are of the same order as capillary length scales, the timescales associated with these capillary waves travelling from the top of the liquid sheet to the crater bottom are comparable to the timescales associated with crater retraction resulting purely due to its gravitational potential. As a result, the two mechanisms often act together and play an important role in the collapse of a crater, namely in accelerating. the collapse. ${ }^{33}$ In the experiments with oil drops presented here, the capillary waves are damped due to higher viscosity of the drop liquid $^{34}$ as they travel towards the crater bottom. The wave convergence and the liquid components' mutual immiscibility makes the drop liquid accumulate at the bottom of the crater. The crater shapes that result due to capillary waves travelling along the initially smooth crater surfaces are shown in Fig. 2b-d and $3 \mathrm{a}-\mathrm{c}$. The presence of a viscous immiscible liquid at the center of the crater prevents a singular collapse of the air cavity, ${ }^{35,36}$ mitigating 'regular' bubble entrainment. ${ }^{34,37}$

When a drop of higher viscosity (200 cSt, Fig. 2e and 3c) is allowed to fall into water, it spreads to a smaller extent than drops of lower viscosities impacted at similar Weber numbers. The importance of viscosity can also be seen by comparing the capillary $\left(\sim D_{0} \sigma\right)$ and drag $\left(\mu D_{0} v\right)$ forces (see respective Ca) from the experiments shown in the Fig. 2 and 3. In our experiments, the value of the Capillary number changes from $\mathrm{Ca} \sim \mathcal{O}\left(10^{-2}\right)$ for an impacting water droplet to $\mathrm{Ca} \sim \mathcal{O}(10)$ for the highest viscosity of oil. Therefore in the latter case, viscosity can significantly damp the deformation of the impacting droplet. The extent to which the drop spreads can have very interesting consequences for oil entrainment. A new oil-water interface with a different surface tension is created along those parts of crater walls where the drop is in contact with water. This 'oilwetted' area is surrounded by a 'non-wetted' water-air interface, which has a higher surface tension than the oil-water interface it surrounds. ${ }^{21}$ As a result, capillary waves occuring on this water-air interface move with a higher velocity than those that occur along oil-water interface. As with drops of lower viscosities (Fig. 2b-d and 3b-c), these waves are damped as they approach the oil-air interface nearer to the crater bottom. As the waves converge, they cause the oil-bulk to collect at the bottom, while the air cavity behind continues to collapse. In certain configurations (such as those shown in Fig. 2e and 3c) during this collapse, the air cavity is lined with an oil layer on the front side. Since the air cavity typically collapses via a 'necking' mechanism, this results in a small air bubble pinching off and getting entrained in the oil phase. Eventually, the oil phase detaches completely from the air-interface, leaving us with a doubly entrained bubble - a small air bubble entrained in a viscous oil drop, which is in turn entrained in the water bulk. A similar type of entrainment has been reported for a reactive drop-pool pair. ${ }^{38}$ Here, however, we show that the same type of double-bubble entrainment can also occur using a pair of immiscible, mutually non-reactive liquids.

\section{Maximum depth of crater}

We find the crater depth $H$ and radius $R$ from experimental images by tracking its perimeter. Henceforth, all length scales discussed are non-dimensionalised with the respective initial 


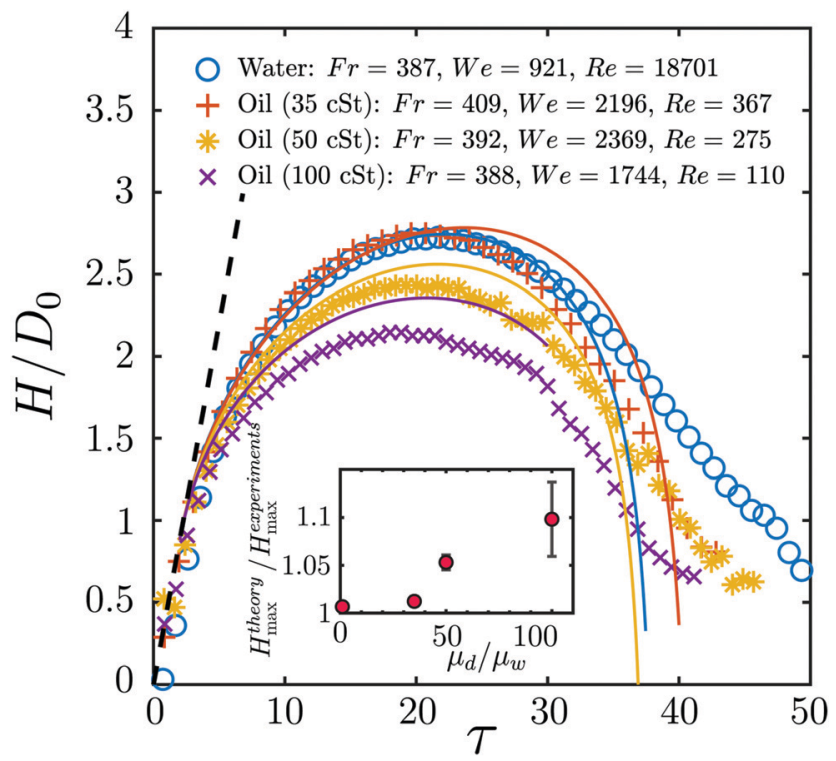

Fig. 4 A comparison of time series of experimentally determined crater depths (data points) with those obtained from Bisighini et al. ${ }^{23}$ model (solid lines with corresponding colour). Only 1 -in-4 data points is shown in the experimental time-series for $H_{\max }$ for clarity. The crater constants $\alpha_{0}$ used to calculate the curves from eqn (3) and (4) are 0.962 for water, 0.958 for 35 cSt silicone oil, 0.779 for 50 cSt silicone oil and 0.648 for 100 cSt silicone oil. The dashed line in the left panel corresponds to $H / D_{0}=0.44 \tau$. We find that the model overpredicts $H_{\text {max }}$ as the impacting drops are made more viscous, which is quantitatively shown in the inset.

drop size $D_{0}$, and the timescales are non-dimensionalised with $v / D_{0}$, such that non-dimensional time $\tau \equiv v t / D_{0}$. The timeseries for the crater depths $H$ from some experiments with $\mathrm{Fr} \approx 400$ are shown in Fig. 4. The principal difference in the behaviours shown by different experiments lie in their approach to the point of crater's maximum depth. This appears to result from differences in viscosities of the drops, the more viscous ones having lower a Re. In experiments with small Re, the capillary waves along the crater walls can travel faster than the penetration speed of the crater itself, and can pose challenge in tracking the crater's boundaries. The waves' influence on the crater's depth can be seen in time series of $50 \mathrm{cSt}$ and $100 \mathrm{cSt}$ drops between $\tau=10-15$ in Fig. 4 . They converge at the crater bottom and cause the bottom tip to vary around the point of maximum depth. The maximum crater depths $H_{\max }$, and the time taken to reach this depth, $\tau_{\max }$ are found from such experimental time series.

\subsection{Heuristic potential flow model}

Here we describe a model to quantitatively describe the temporal evolution of the crater depths. It was reported by Engel $e t$ al. ${ }^{22}$ that, as in our experiments of impacting water drop on the pool, the crater formed in the target pool initially has a flat bottom. Soon it reaches a roughly-hemispherical shape, and a flow is established in the pool, which 'appeared to be associated with the existence of both a radial and a tangential velocity in the liquid'. ${ }^{22}$ Bisighini et al. ${ }^{23}$ used these observations to model crater dynamics caused after the impact of a drop on a pool of the same liquid. They modelled the resulting flow in the pool as a linear superposition of flow potentials caused by flow past a sphere and a radially expanding sphere. The sphere, centred on $O^{\prime}$, translates along the vertical coordinate $\zeta$. Its velocity of translation is denoted by $\dot{\zeta}$. Similarly, $\alpha$ is the radial coordinate, also centred on $O^{\prime}$, along which the sphere expands with a speed expressed as $\dot{\alpha}$. These quantities are described in Fig. 5 and are non-dimensionalised by $D_{0}$. The final equations of motion for the sphere are derived by balancing stresses at the crater interface for a thinly spread drop:

$$
\begin{gathered}
\ddot{\alpha}=-\frac{3}{2} \frac{\dot{\alpha}^{2}}{\alpha}-\frac{2}{\alpha^{2} \mathrm{We}}-\frac{1}{\operatorname{Fr}} \frac{\zeta}{\alpha}+\frac{7}{4} \frac{\dot{\zeta}^{2}}{\alpha}-\frac{4 \dot{\alpha}}{\alpha^{2} \operatorname{Re}} \text {, and } \\
\ddot{\zeta}=-3 \frac{\dot{\alpha} \dot{\zeta}}{\alpha}-\frac{9}{2} \frac{\dot{\zeta}^{2}}{\alpha}-\frac{2}{\operatorname{Fr}}-\frac{12 \dot{\zeta}}{\alpha^{2} \operatorname{Re}},
\end{gathered}
$$

where the full derivation is omitted to avoid repetition of the original contained in ref. 23 The viscous effects in pool liquid are included in the above system of equations via the terms $4 \dot{\alpha} / \alpha^{2} \operatorname{Re}$ and $12 \dot{\zeta} / \alpha^{2} \operatorname{Re}$ by considering the shear stresses in a thin layer of fluid along the free surface of the crater. The normal stress jump from viscous effects is ignored as the relevant layer of fluid is very thin. In our experiments, where there is a thin layer of another liquid (silicone oil) on the other side of the crater surface, the shear stresses are the same on either side of the oil-water interface in the crater. ${ }^{39}$ Therefore, the terms mentioned above can equally well be used to incorporate the viscous dissipation in a layer of oil which is thinly spread over the crater surface. We only concern with viscous dissipation in the oil phase as it is orders of magnitude greater than the dissipation in surrounding thin layers in water.

To this end, the system of eqn (3) and (4) can be solved numerically by supplementing with appropriate initial conditions and constants. Bisighini et al. $^{23}$ calculate the crater's radial speed of expansion $\dot{\alpha} \approx 0.17$ and speed of sphere's translation $\dot{\zeta} \approx 0.27$, for the initial stage of drop penetration $(\tau<2)$. Their calculation leads to a simple asymptotic scaling of $H / D_{0}=0.44 \tau$

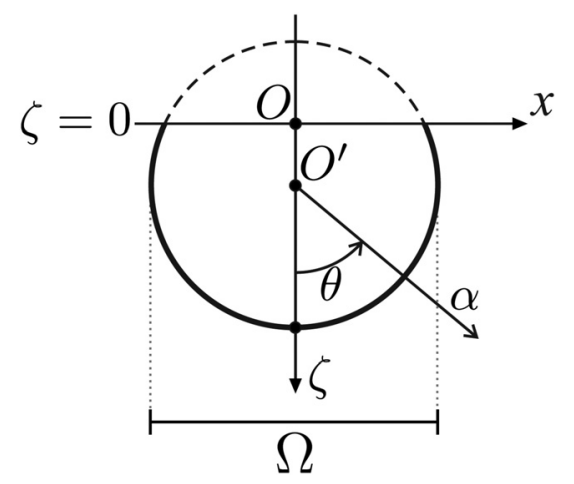

Fig. 5 System of coordinates defined for the model contained in eqn (3) and (4). The system models a combination of flows caused by a sphere centred on $O^{\prime}$, which translates downwards along $\zeta$ and expands along $\alpha$. $\Omega$ is the diameter of the expanding crater. The original undisturbed free surface lies at $\zeta=0$. The figure has been adapted from ref. 23 . 
for small times. These values lead us to the following set of initial conditions

$$
\dot{\alpha} \approx 0.17, \quad \dot{\zeta} \approx 0.27, \quad \alpha \approx \alpha_{0}+0.17 \tau \text { and } \zeta \approx-\alpha_{0}+0.27 \tau,
$$

which are required to solve the system described by eqn (3) and (4). The physical interpretation of $\alpha_{0}$ is the initial crater depth at $\tau=2$. Beyond $\tau=2$, the initially rapid growth of crater depth with a speed of $0.44 \tau$ ceases to hold, and the drop liquid plays an increasingly greater role in determining the crater depth. The numerical values for $\alpha_{0}$ are found by fitting the experimentally determined crater diameter to the following expression, based on geometrical reasons:

$$
\Omega=2 \sqrt{\alpha^{2}-\zeta^{2}} \approx 2 \sqrt{\left(\alpha_{0}+0.17 \tau\right)^{2}-\left(0.27 \tau-\alpha_{0}\right)^{2}} .
$$

A series of results computed using this model are compared with experimental data in Fig. 4. The initial, inertia dominated stage, following the asymptotic solution of Bisighini et al. ${ }^{23}$ matches well with our observations. Such dynamics suggest the negligible influence of drop viscosity in the early stages. We find from Fig. 4 that given a Froude number of impact, higher drop viscosities result in the formation of shallower craters in the target pool. A comparison between the maximum depths predicted by the model and those measured in experiments is shown in the inset of Fig. 4. We find that the model's predictions exceed those measured from experiment. With increasing viscosity of the drop, the disagreement between model and experiments increases. This gives an important insight into the limitations of the model, which arises from how it accounts for viscous dissipation. We observe from the experiments that as increasingly viscous drops are impacted on the pool, they form increasingly thicker layers along the crater surface. However, the modelling of viscous losses in the system is limited to dissipation in a thinly spread layer of oil. It is unable to account for dissipation in these thicker layers of drop liquid, thereby overpredicting the crater depths, specially for more viscous drops.

In the later stages ( $\tau>20$ in Fig. 4 ), we find that the collapsing phase of the crater's bottom is consistently found to be steeper in the model than in the experiments. It can be seen in experimental images in Fig. 2 and 3, that regardless of whether a water drop or an oil drop impacts, there exist capillary waves that travel along the crater surface. As the crater begins to collapse due to its potential energy, these capillary waves travel in the opposite direction and effectively counter the buoyancy, thereby retarding the collapse of crater. The collapse is further slowed down in cases where a viscous drop impacts and creates the crater. Recall from Section 3.1 how the drop accumulates at the crater bottom during the retraction phase. Such an accumulation of a viscous fluid at the crater bottom would damp the capillary waves, but would also inevitably interfere with bouyancy-driven retraction of the crater and slow it down.

\subsection{Inertial and viscous effects in determining}

At $\tau_{\max }$, the maximum depth of crater, $H_{\max } / D_{0}$ can be modelled by balancing initial drop energy with the potential energy associated with the crater at its maximum size. Assuming the crater to have a hemispherical shape at the time of its maximum depth, and approximating a vanishing velocity field in the liquid at $\tau_{\max }$, a relation between the maximum crater depth and Froude number can be derived: $\mathbf{1}^{1,24,27}$

$$
H_{\max } / D_{0} \sim\left(\mathrm{Fr} / \rho^{\prime}\right)^{1 / 4}
$$

where $\rho^{\prime}$ is the density ratio between water and drop liquid. We show in Fig. 6 , that in the vicinity of $\tau_{\max }$, the crater depth $H_{\max }$ and radius $R_{\tau_{\max }}$ are approximately equal. Thus the crater shape at $\tau_{\max }$ is roughly hemispherical, which allows us to test this scaling in Fig. 7a.

We find in Fig. 7a that our results for $H_{\max }$, along with previous works ${ }^{22,24,40}$ appear to follow the scaling shown in eqn (7). We conclude that for the drop parameters included in the figure, the inertia of the pool liquid plays a qualitatively similar role in determining the maximum depth of craters created by impacting oil droplets. From Fig. 7b, we see that for a given $\mathrm{Fr} / \rho^{\prime}$, that the greater the drop viscosity, the smaller the $H_{\max }$ of the crater. It is clear from Fig. 2 and 3 that craters formed by drops of up to $100 \mathrm{cSt}$ resemble the hemispherical craters formed by water drops. Oils with higher viscosities (here, $200 \mathrm{cSt}$ ) do not create such craters - they do not spread thinly over the crater surface, and consequently, do not satisfy the assumptions inherent in the model contained in eqn (3) and (4), and the scaling expressed in eqn (7). Thus, we only consider the data from oil viscosities up to $100 \mathrm{cSt}$ in the following analysis.

Another interesting observation to be made from Fig. 7b is that the data from different drop viscosities have small differences in slope, exhibiting a small but systematic deviation from the $\left(\mathrm{Fr} / \rho^{\prime}\right)^{1 / 4}$ scaling. We suspect this to be a result of the assumptions that are implicit in the derivation of this scaling. Additionally, this is also suspected to result from the so far un-investigated influence of viscosity on $H_{\max }$. The influence of $\mu$ on $H_{\max }$ can be more

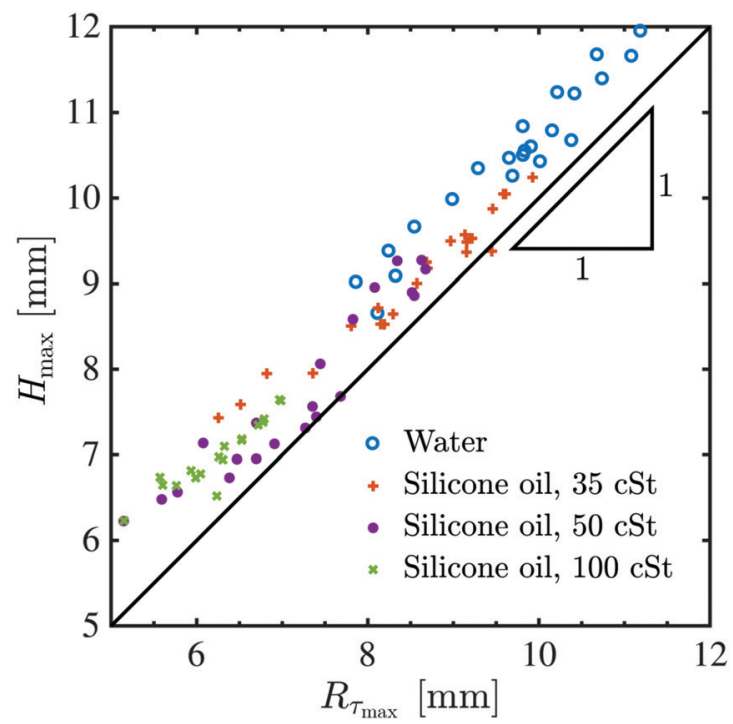

Fig. 6 Comparison between the a crater's depth and radius at $\tau_{\max }$. $H_{\max }$ and $R_{\tau_{\max }}$ are found to be roughly equal in size, which shows that the crater is approximately hemispherical in shape. Along the black line, $H_{\max }=R_{\tau_{\max }}$. 
(a)
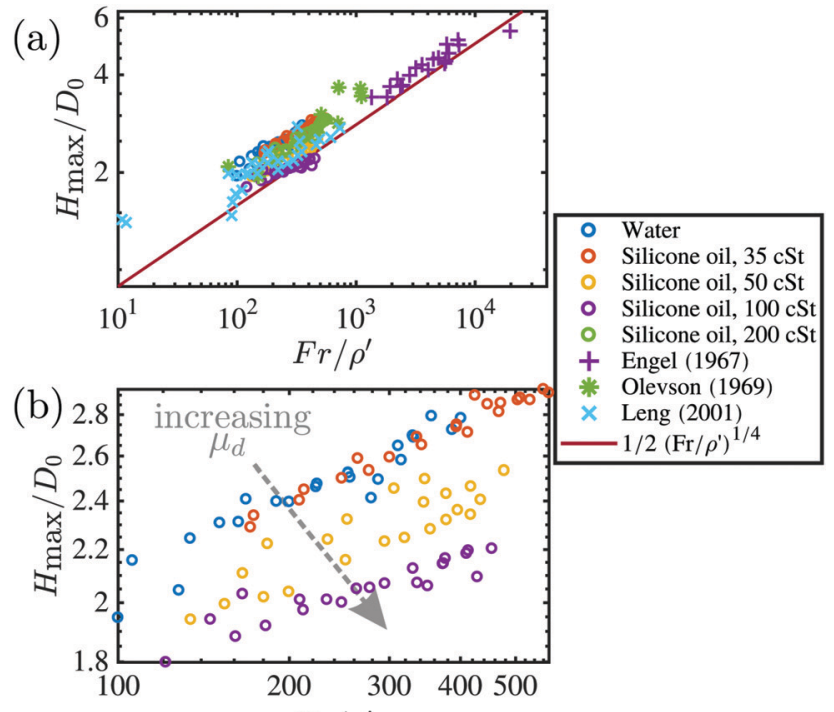

(c)

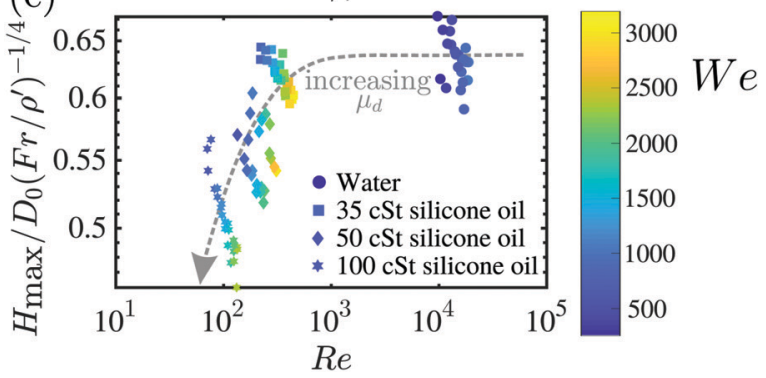

Fig. 7 (a) Maximum depth of craters plotted against Froude number. $H_{\text {max }} / D_{0}$ resulting from oil drops impacting water roughly follow the scaling $\left(\mathrm{Fr} / \rho^{\prime}\right)^{1 / 4}$. (b) A zoomed-in region of the plot in (a), and contains only the new results. The data here show a systematic trend in $H_{\max } / D_{0} v s$. $\left(\mathrm{Fr} / \rho^{\prime}\right)$ as a result of change in drop viscosity. Note the shared legend between subfigures (a) and (b). (c) The variation of Froude-scalingcompensated $H_{\text {max }} / D_{0}$ with $R e$ is plotted.

systematically isolated and checked by compensating the $H_{\max } / D_{0}$ results by $\left(\mathrm{Fr} / \rho^{\prime}\right)^{1 / 4}$ scaling, and using another non-dimensional number, such as Re, which can capture the change in drop viscosity. Any deviations from $H_{\max } / D_{0} \sim\left(\mathrm{Fr} / \rho^{\prime}\right)^{1 / 4}$ scaling would expectedly continue to be visible in such a compensated plot. This is done in Fig. 7c, where the Froude-scaling is compensated-for in the measured crater-depths, and plotted against Re. This enables us to separate the roles played by $\mathrm{Fr}$ and Re in determining the crater depths.

From the results shown in Fig. 7c, we recover the same trend of $H_{\max }$ with varying $\mu$ as shown in the adjoining figure, where $H_{\max }$ are seen to be smaller for larger $\mu$. This behaviour is also in agreement with our observation in earlier sections (specifically Fig. 4 and 6) where a larger drop viscosity was seen to result in shallower craters. A more viscous drop results in a shallower crater because there is larger amount of viscous dissipation in the drop itself, leaving lesser energy to be imparted to the pool liquid. From Fig. $7 \mathrm{c}$ we also see that impacts with a wide range of Weber numbers are distributed across a wide range of the shown parameter space, suggesting a weak correlation, if any, between We and $H_{\max }$.

\section{Fingers along rim}

The impact of an immiscible oil drop initially results in a typical crown splash above the initially stationary pool surface, which is accompanied by outwards-moving capillary waves, a growing crater and a wave swell. The splash crown consists of both drop and pool liquids, and depending on the drop's impact velocity, viscosity and interfacial surface tension with pool liquid, it might spread over the crater surface to a varying extent. The presence of drop liquid in the crown was recently also reported in the works by Che \& Matar ${ }^{19}$ and Shaikh et al. ${ }^{20}$ both of which studied water drop impacts on thin films of oil. Such a crown which is made of both the liquid components was termed a 'compound crown'19 - its outer surface is composed of target pool liquid (in present study, water) and the inner surface is covered with the drop liquid (here, silicone oil). After ejection of tiny-drops by corona, the rim begins to stabilise as the sheet retracts back into the pool. At this stage, the remnant small-amplitude perturbations can still be seen along the compound-crown rim. These perturbations are quickly suppressed by high surface tension in the water phase but not so in the oil phase. As a result, the perimeter of retracting drop remains deformed - and these deformations are not smoothened out by surface tension as in water.

As the crater retracts, the small deformations along oil-drop rim stretch into fingers, which can be seen due to a small difference in the two liquids' refractive indices. We recorded the experiments from the bottom (shown in Fig. 8; also see ESI $\dagger$ for an example of such an experiment) and counted the number of fingers $N_{\mathrm{f}}$ observable in a number of experiments. See also Fig. 10 in the Appendix where the same phenomena is shown from a different angle than bottom-view. In the experiment shown in Fig. 10, a small amount of milk powder was mixed with water to enhance the image contrast.

The number of fingers observed in each experiment was found to be closely correlated to the number of observable peaks along the rim of a retracting corona and always within a certain range of 10-15 as shown in Fig. 9. This was also the case in the recent paper of Shaikh $e t a .^{20}$ for drop impacts on thin films, where the number of fingers are always within a range of $11-19,{ }^{41}$ independent of the non-dimensional groups. We therefore looked at the correlations in which the number of corona fingers is predicted. Marmanis and Thoroddsen ${ }^{42}$ proposed the so-called impact Reynolds number, $\mathrm{Re}_{\mathrm{I}}$, based on the thickness of the crown walls $\delta \approx\left(\mu_{\mathrm{d}} T / \rho\right)^{1 / 2}$, where $T$ is the natural oscillation period of the droplet (as the deformation time scale). They eventually suggested that the number of fingers should be correlated as,

$$
N_{\mathrm{f}}=\alpha \operatorname{Re}_{\mathrm{I}}^{\beta}, \quad \text { with } \quad \operatorname{Re}_{\mathrm{I}}=\mathrm{We}^{1 / 4} \mathrm{Re}^{1 / 2},
$$

where $\alpha$ and $\beta$ are fitting parameters. Marmanis and Thoroddsen ${ }^{42}$ found $\beta=3 / 4$ for impact of droplets. Katsuragi ${ }^{43}$ found $\beta=1 / 3$ for impact of solid spheres on viscous fluids. In our experiments, the values of $\mathrm{Re}_{\mathrm{I}}$ only varies within the range of 50-90. Such a range is so narrow that we can not expect much change in the number of fingers. This can be confirmed by looking at the data of Marmanis and Thoroddsen ${ }^{42}$ and Katsuragi ${ }^{43}$ where $N_{\mathrm{f}} \approx 10$ for this range. 

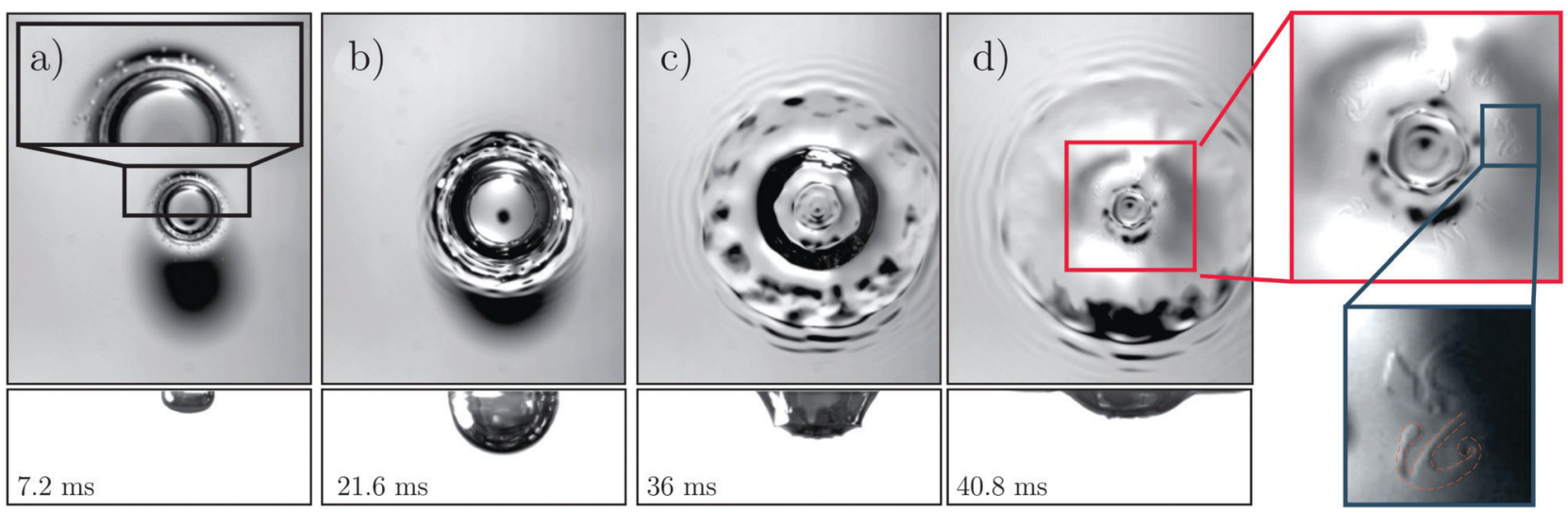

Fig. 8 Impact of a $50 \mathrm{cSt}$ silicone oil, $3.7 \mathrm{~mm}$ in diameter, at $4.22 \mathrm{~m} \mathrm{~s}^{-1}$ on a pool of stationary water. Top and bottom pictures correspond to bottom and side views, respectively. The corresponding non-dimensional numbers are $\mathrm{We}=3028, \mathrm{Re}=175, \mathrm{Fr}=491, \mathrm{a} \mathrm{Ca}=17.3$. Upon impact, splashing occurs (highlighted in panel a) and a crater forms and grows (b). The crater eventually begins to retract (c and d) while capillary waves are formed at the crater and surface of the bath. We observe the fingers at this point (magnified in panel d) when the crater is retracting and forming a Worthington jet on the surface (not shown here).

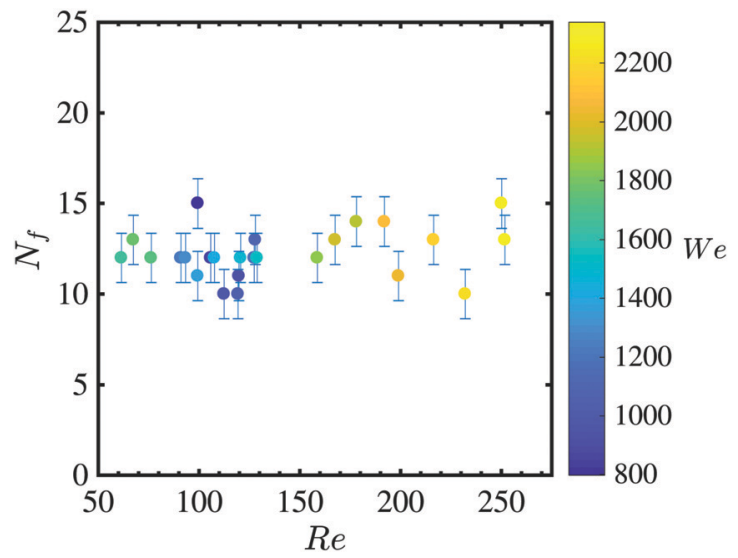

Fig. 9 Number of fingers from a series of experiments over a range of Re and We, where $N_{f}$ were counted as shown in Fig. 8. Error bars are the standard deviation over the shown data.

Another approach to find the number of fingers is based on the Rayleigh-Taylor instability, ${ }^{44}$ assuming the cylindrical sheet of the liquid is accelerating in air (also see Zhang et al. ${ }^{45}$ ). Linear stability analysis leads to a dominant wave number of:

$$
\kappa=\left(\rho_{\mathrm{w}} v^{2} / 3 D \sigma\right)^{1 / 2} \equiv \frac{1}{3^{1 / 2} D} \mathrm{We}^{1 / 2},
$$

where, $v^{2} / D$ is the characteristic acceleration and we ignore the density of air. Katsuragi ${ }^{43}$ assumed that the instability occurs on a perimeter of a circle of diameter $D_{\mathrm{c}}=1 / 2 D \mathrm{Re}^{1 / 4}$, where the diameter is the maximum spreading diameter of droplet upon impact on hard surfaces. Finally, the following expression for the number of fingers was obtained:

$$
N_{f}=\frac{1}{48^{1 / 2}} \mathrm{We}^{1 / 2} \mathrm{Re}^{1 / 4} .
$$

Note, in comparison with eqn (8), the equation above has different scalings for We and Re and has no fitting parameters.
Nevertheless, similar to the previous correlation, for our experiments, the number of fingers using eqn (10) also only varies within the range of 11-22. The analyses above suggest the unchanged values of $N_{\mathrm{f}}$ (shown in Fig. 9) are most likely only due to limited parameter range available in the present experiments.

In the later stage of crater collapse, the retraction accelerates due to combined effects of gravitational retraction and capillary wave focussing. This causes the fingers to stretch significantly, while their tips may undergo tip-splitting. This makes the tips of the fingers appear like mushrooms (see the magnified views in panel $d$ of Fig. 8). Note that since this stage of crater retraction is associated with rapid flow focussing, this observed tip-splitting may be ascribed to a shear instability due to a large jump in shear velocities occurring at the oil-water interface. Alternately, it could also be an outcome of viscous-fingering of the type produced in Taylor-Saffman instabilities, wherein such tip-splitting is a known mechanism of instability growth.

\section{Conclusions}

Here we reported the results from experiments studying the impacts of viscous immiscible oil drop on a deep pool of water. We use a range of viscosities of the oil, which, due to the added effect of immiscibility, result in a variety of interesting phenomena. An object of major interest in drop impacts is often the crater formed in the target pool. We find that the crater dynamics depend crucially on how the impinging drop behaves after impacting on the free surface. If the impinging drop spreads over the entirety of the crater surface, as is usually observed when the drop-target liquids are the same, the primary effect of increasing viscosity of the drop is that the crater is shallower. As the drop viscosity is increased further, it is unable to spread appreciably over the crater surface due to larger viscous dissipation. The drop's interaction with capillary waves makes the drop liquid accumulate at the crater bottom, and may 


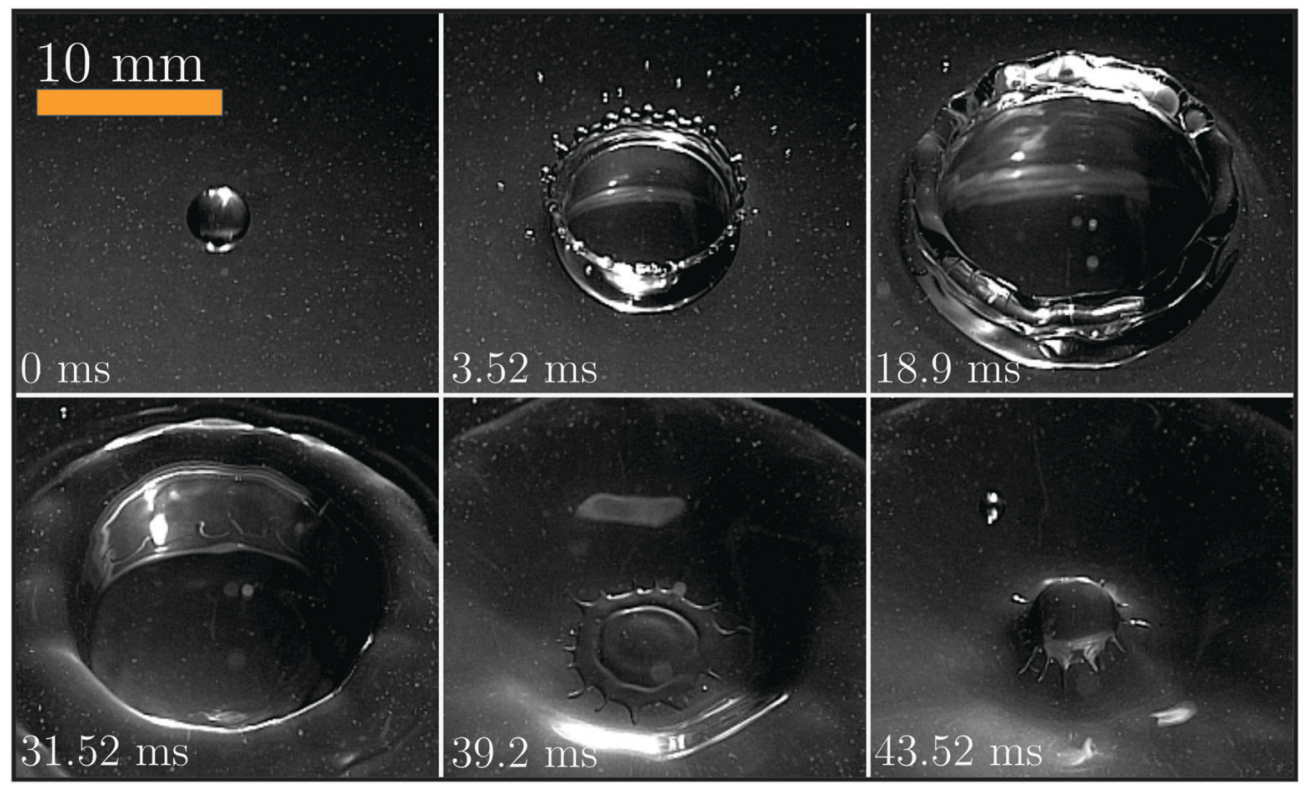

Fig. 10 A $3.46 \mathrm{~mm}$ wide drop of $100 \mathrm{cSt}$ silicone oil impacting on a pool of water at $3.5 \mathrm{~m} \mathrm{~s}^{-1}$. A small amount of milk powder was added to water to aid visualisation. The impacting drop spreads throughout the crater, whose edges destabilise leading to the well known splash-crown ( $3.52 \mathrm{~ms}$ ). In the retraction stage (here $31.52 \mathrm{~ms}$ onwards), the perturbations along the crown-rim continue to exist along the retracting drop rim and get stretched into the fingers as shown at $39.2 \mathrm{~ms}$.

result in its complete detachment from the water surface. In some cases, we also obtain double-entrainment of an air bubble within an entrained oil droplet. deeper understanding of this phenomenon can be of use in controlling various capsuleformation and encapsulation processes, particularly in pharmaceutical settings. ${ }^{46-49}$

We employ the model proposed by Bisighini et al. ${ }^{23}$ to model the crater growth, where we include the viscous dissipation in the drop based on shear stress balance arguments. We find that the model works well for the early stages. It overpredicts the crater depths at the point of their maximum depth and predicts a faster collapse of the crater than is measured. The sources of disagreement between the model and experiments were identified and discussed.

The impact of an immiscible drop into a pool of another liquid results in the formation of a compound splash sheet which consists of both the liquids risen above the initially calm pool surface. The rim of the sheet becomes hydrodynamically unstable and ejects tiny droplets. The deformations along the edge of the sheet during this stage are imposed on both the liquids which compose the crown. In our experiments, the perturbations along drop rim persist beyond the crater retraction stage, and get stretched into fingers. Such finger formation in the crown has been reported during water drop impacting on thin oil film experiments. ${ }^{19,20}$ We report that the same phenomenon occurs when an immiscible oil drop is impacts on a deep pool of water. A detailed understanding of this may give insights into fragmentation processes found in nature and industry. In this way, one may find the means to control the size distribution of daughter droplets produced via such violent fragmentation processes. ${ }^{17,20}$

\section{Conflicts of interest}

There are no conflicts to declare.

\section{Appendix: imaging fingers from top}

To have a better understanding on the mechanism of the finger formation, we also performed the experiments in a slightly modified experimental setup, using a high speed camera to record from the top. However, it was often difficult to identify the oil fingers in these experiments due to very similar refractive indices of the two liquids. ${ }^{50}$ We attempted to improve the contrast between the two liquids by colouring the oil with a fat soluble dye up to the point of its saturation. However, given how thinly the oil layer is spread around the point of $\tau_{\max }$, this method failed to provide a sufficiently large contrast difference. Thus, we resorted to colouring the water instead, and a small amount of milk powder was added to the water bulk to get a better contrast between the two phases. Fig. 10 shows a series of pictures in the setting explained above. See also ESI $\dagger$ for a video of the experiment discussed here. The images obtained provide a conclusive evidence for the presence of fingering. The milk powder slightly modified water properties, which resulted in a greater number of fingers than typically seen, and the mushroomlike structures shown in Fig. 8 could not develop in the shown experiment.

\section{Acknowledgements}

We would like to acknowledge funding from SLING (project number P14-10.1) which is (partly) financed by the Netherlands 
Organisation for Scientific Research (NWO). We would also like to thank V. Sanjay for helpful comments on an earlier version of the manuscript and J. Marston, A. Prosperetti and R. Krechetnikov for helpful discussion on the fingers results.

\section{References}

1 A. Prosperetti and H. N. Oğuz, Annu. Rev. Fluid Mech., 1993, 25, 577-602.

2 M. Rein, Fluid Dyn. Res., 1993, 12, 61-93.

3 A. Worthington, A study of splashes, Longmans, Green, London, 1908, p. 129.

4 O. G. Engel, J. Appl. Phys., 1966, 37, 1798-1808.

5 H. C. Pumphrey and P. A. Elmore, J. Fluid Mech., 1990, 220, 539-567.

6 A. Yarin, Annu. Rev. Fluid Mech., 2006, 38, 159-192.

7 C. Josserand and S. Thoroddsen, Annu. Rev. Fluid Mech., 2016, 48, 365-391.

8 A. L. Yarin, I. V. Roisman and C. Tropea, Collision phenomena in liquids and solids, Cambridge University Press, 2017.

9 C. Clanet, C. Béguin, D. Richard and D. Quéré, J. Fluid Mech., 2004, 517, 199-208.

10 T. T. Truscott, B. P. Epps and J. Belden, Annu. Rev. Fluid Mech., 2014, 46, 355-378.

11 R. C. Hurd, J. Belden, M. A. Jandron, D. T. Fanning, A. F. Bower and T. T. Truscott, J. Fluid Mech., 2017, 824, 912-930.

12 M. Jalaal, D. Kemper and D. Lohse, J. Fluid Mech., 2019, 864, 596-613.

13 D. W. Murphy, C. Li, V. d'Albignac, D. Morra and J. Katz, J. Fluid Mech., 2015, 780, 536-577.

14 Y. Wu, Oil Spill Impacts: Taxonomic and Ontological Approaches, CRC Press, 2016.

15 T. W. Walker, A. N. Logia and G. G. Fuller, Exp. Fluids, 2015, 56, 106.

16 V. Sharma, M. Szymusiak, H. Shen, L. C. Nitsche and Y. Liu, Langmuir, 2012, 28, 729-735.

17 H. Lhuissier, C. Sun, A. Prosperetti and D. Lohse, Phys. Rev. Lett., 2013, 110, 264503.

18 T. Fujimatsu, H. Fujita, M. Hirota and O. Okada, J. Colloid Interface Sci., 2003, 264, 212-220.

19 Z. Che and O. K. Matar, Soft Matter, 2018, 14, 1540-1551.

20 S. Shaikh, G. Toyofuku, R. Hoang and J. Marston, Exp. Fluids, 2018, 59, 7.

21 P. Than, L. Preziosi, D. Josephl and M. Arney, J. Colloid Interface Sci., 1988, 124, 552-559.

22 O. G. Engel, J. Appl. Phys., 1967, 38, 3935-3940.

23 A. Bisighini, G. E. Cossali, C. Tropea and I. V. Roisman, Phys. Rev. E: Stat., Nonlinear, Soft Matter Phys., 2010, 82, 036319.

24 L. J. Leng, J. Fluid Mech., 2001, 427, 73-105.
25 J. B. Lee, D. Derome, A. Dolatabadi and J. Carmeliet, Langmuir, 2016, 32, 1279-1288.

26 S. Wildeman, C. W. Visser, C. Sun and D. Lohse, J. Fluid Mech., 2016, 805, 636-655.

27 A. I. Fedorchenko and A.-B. Wang, Phys. Fluids, 2004, 16, 1349-1365.

28 G.-J. Michon, C. Josserand and T. Séon, Phys. Rev. Fluids, 2017, 2, 023601.

29 H. N. Oǧuz and A. Prosperetti, J. Fluid Mech., 1990, 219, 143-179.

30 H. N. Oǧuz and A. Prosperetti, J. Fluid Mech., 1991, 228, 417-442.

31 D. Morton, M. Rudman and L. Jong-Leng, Phys. Fluids, 2000, 12, 747-763.

32 W. Bouwhuis, X. Huang, C. U. Chan, P. E. Frommhold, C.-D. Ohl, D. Lohse, J. H. Snoeijer and D. van der Meer, J. Fluid Mech., 2016, 792, 850-868.

33 S. Gekle, A. van der Bos, R. Bergmann, D. van der Meer and D. Lohse, Phys. Rev. Lett., 2008, 100, 084502.

34 Q. Deng, A. Anilkumar and T. Wang, J. Fluid Mech., 2007, 578, 119-138.

35 M. S. Longuet-Higgins, J. Fluid Mech., 1983, 127, 103-121.

36 S. Gekle, J. M. Gordillo, D. van der Meer and D. Lohse, Phys. Rev. Lett., 2009, 102, 034502.

37 B. Ray, G. Biswas and A. Sharma, Phys. Fluids, 2012, 24, 082108.

38 Q. Deng, A. Anilkumar and T. Wang, J. Colloid Interface Sci., 2009, 333, 523-532.

39 S. S. Pegler and M. G. Worster, J. Fluid Mech., 2012, 696, 152-174.

40 K. Olevson, US Geological Survey Professional Paper, 1969, p. 189.

41 Private communication with Jeremy Marston.

42 H. Marmanis and S. T. Thoroddsen, Phys. Fluids, 1996, 8, 1344-1346.

43 H. Katsuragi, EPJ Web of Conferences, 2015, p. 02032.

$44 \mathrm{~S}$. Chandrasekhar, Hydrodynamic and hydromagnetic stability, Courier Corporation, 2013.

45 L. V. Zhang, P. Brunet, J. Eggers and R. D. Deegan, Phys. Fluids, 2010, 22, 122105.

46 T. Wang, I. Lack, M. Brissová, A. V. Anilkumar, A. Prokop, D. Hunkeler, R. Green, K. Shahrokhi and A. C. Powers, Nat. Biotechnol., 1997, 15, 358.

47 A. V. Anilkumar, I. Lacik and T. G. Wang, Biotechnol. Bioeng., 2001, 75, 581-589.

48 Y. Yeo, O. A. Basaran and K. Park, J. Controlled Release, 2003, 93, 161-173.

49 Y. Yeo, A. U. Chen, O. A. Basaran and K. Park, Pharm. Res., 2004, 21, 1419-1427.

50 Wacker Chemie AG, WACKER AK 100 SILICONE FLUID Technical Data Sheet, Nov 2014. 